\title{
Estrutura da comunidade dos Tintinnina na região do Atol das Rocas (Rio Grande do Norte) e Arquipélago de Fernando de Noronha (Pernambuco)
}

\author{
Eliane Maria de Souza Nogueira ${ }^{1,3}$, Roberto Sassi $^{2}$ \& Tarcísio Alves Cordeiro ${ }^{2}$ \\ ${ }^{1}$ Departamento de Educação, Campus VIII, \\ Universidade do Estado da Bahia - UNEB, \\ Rua do Gangorra, 503, CEP 486000-000, Paulo Afonso, BA, Brasil \\ ${ }^{2}$ Universidade Federal da Paraíba - UFPB, \\ Centro de Ciências Exatas e da Natureza-CCEN, \\ Departamento de Sistemática e Ecologia - DSE, \\ Núcleo de Estudos e Pesquisas de Recursos do Mar - NEPREMAR, \\ Pós-Graduação em Desenvolvimento e Meio Ambiente - PRODEMA, \\ CEP 58049-900, João Pessoa, PB, Brasil, \\ e-mail: sassi_rs@yahoo.com.br,tacordeiro@gmail.com \\ ${ }^{3}$ Autor para correspondência: Eliane Maria de Souza Nogueira, e-mail: emsnogueira@gmail.com.br
}

NOGUEIRA, E.M.S., SASSI, R. \& CORDEIRO, T.A. 2008. Community structure of Tintinnina near the Atol das Rocas Island (Rio Grande do Norte) and Fernando de Noronha Archipelago (Pernambuco). Biota Neotrop. 8(3): http://www.biotaneotropica.org.br/v8n3/en/abstract?article+bn01708032008.

\begin{abstract}
Tintinnina occurring near the Atol das Rocas Island and Fernando de Noronha Archipelago (northeastern Brazil) were studied using plankton samples gathered from 13 to 30 March, 1999, in 27 fixed oceanic stations. The study sought to characterize the spatial distribution of these ciliates in the area and to examine parameters such as diversity and equitability that define the community structure. The material was collected by vertical hauls from $200 \mathrm{~m}$ to the surface using a $20 \mu \mathrm{m}$ mesh plankton net, preserved in $4 \%$ neutral formalin, and examined using an inverted microscope. The highest density of individuals was found in station $26\left(122 \mathrm{ind}^{-3} \mathrm{~m}^{-3}\right.$, which is situated very close to Fernando de Noronha; but high densities were also registered in stations $1\left(82.7\right.$ ind. $\left.\mathrm{m}^{-3}\right)$ and $2\left(74.7\right.$ ind. $\left.\mathrm{m}^{-3}\right)$, which were situated in the most distant areas, as well as in station $5\left(80.7\right.$ ind. $\left.\mathrm{m}^{-3}\right)$ close to the Atol das Rocas Island. Eutintinnus fraknoi (Daday) Kofoid \& Campbell, 1929 was the most abundant species, attaining $57.8 \%$ of dominance in station 4 . The index of specific diversity fluctuated from high $(27 \%$ of the samples with values higher than 3 bits.ind ${ }^{-1}$ ) to medium ( $63 \%$ of the samples higher than 2 bits.ind $\left.^{-1}\right)$, while the equitability fluctuated between 0.6 and 1.0. The low densities of Tintinnina observed in the material collected confirm the oligotrophic condition of that region.
\end{abstract}

Keywords: oceanic plankton, spatial distribution, Western South Atlantic, microzooplankton, northeast Brazil.

NOGUEIRA, E.M.S., SASSI, R. \& CORDEIRO, T.A. 2008. Estrutura da comunidade dos Tintinnina na região do Atol das Rocas (Rio Grande do Norte) e Arquipélago de Fernando de Noronha (Pernambuco). Biota Neotrop. 8(3): http://www.biotaneotropica.org.br/v8n3/pt/abstract?article+bn01708032008.

Resumo: Os Tintinnina que ocorrem em águas próximas ao Atol das Rocas e Arquipélago de Fernando de Noronha (Nordeste do Brasil) foram estudados usando amostras de plâncton coletadas entre 13 e 30 de março de 1999 em 27 estações oceânicas. O estudo procurou caracterizar a distribuição espacial desses ciliados na área e examinar parâmetros como a diversidade e eqüitabilidade que definem a estrutura da comunidade. O material foi coletado por arrastos verticais desde $200 \mathrm{~m}$ de profundidade até a superfície, usando uma rede de plâncton de $20 \mu \mathrm{m}$ de abertura de malhas, preservado em formol neutro a 4\%, e examinado em um microscópio invertido. A maior densidade de indivíduos foi encontrada na estação 26 (122 ind. $\left.\mathrm{m}^{-3}\right)$, situada nas proximidades de Fernando de Noronha; mas altas densidades também foram registradas nas estações $1\left(82,7\right.$ ind. $\left.\mathrm{m}^{-3}\right)$ e $2\left(74,7\right.$ ind. $\left.\mathrm{m}^{-3}\right)$, situadas em áreas mais distantes, bem como na estação $5\left(80,7\right.$ ind.m $\left.{ }^{-3}\right)$ próxima do Atol das Rocas. Eutintinnus fraknoi (Daday) Kofoid \& Campbell, 1929 foi a espécie mais abundante, atingindo 57,8\% de dominância na estação 4. O índice de diversidade específica variou de alto (27\% das amostras com valores maiores que 3 bits.ind ${ }^{-1}$ ) a médio ( $63 \%$ das amostras com valores maiores que 2 bits.ind $^{-1}$ ), enquanto a eqüitabilidade variou entre 0,6 e 1,0. As baixas densidades de Tintinnina observadas no material coletado confirmam a condição oligotrófica daquela região.

Palavras-chave: plâncton oceânico, distribuição espacial, Atlântico Sul Ocidental, microzooplâncton, Nordeste do Brasil. 


\section{Introdução}

As informações disponíveis sobre o microplâncton de águas tropicais do Atlântico Sul Ocidental são ainda muito escassas. Os poucos trabalhos realizados nessa região têm considerado apenas formas com esqueleto rígido ou lórica, como os foraminíferos e os tintiníneos, e abordaram apenas aspectos taxonômicos.

Em se tratando de áreas da plataforma externa e oceânica há uma carência muito grande de outros tipos de estudo, ao contrário do que ocorre em outras regiões oceânicas ao sul do hemisfério sul (Fernandes 1999), particularmente em águas situadas na zona de transição entre a corrente do Brasil e a Corrente das Malvinas, e em águas antárticas e subantárticas, onde diversos estudos enfocando os Tintinnina e outros componentes do microzooplâncton foram realizados. (Balech 1958, 1962, 1971, Souto 1970a, 1972, Balech \& Souto 1980, 1981, Sassi e Melo 1986, 1993, Thompson et al. 1999).

Na região tropical do Atlântico Sul Ocidental a ênfase tem sido o estudo de áreas costeiras e estuarinas (Sassi \& Melo 1986, 1989, Nogueira-Paranhos \& Paranaguá 1991, Sassi et al. 2004), devido à facilidade de acesso e a praticidade para obtenção de amostras. A necessidade de utilização de navios oceanográficos em águas afastadas da costa tem limitado as pesquisas planctônicas nessas áreas, visto que a ocorrência de grandes expedições oceanográficas não acontece com muita freqüência, e poucas vezes são direcionadas ao estudo do plâncton.

Especialmente para a área onde esta pesquisa foi realizada, podem ser citadas as participações dos navios oceanográficos Victor Hensen (Programa JOPS II - Joint Oceanographic Projects; Brazilian German Oceanographic Programme) e Antares (Programa de Avaliação do Potencial Sustentável de Recursos Vivos na Zona Econômica Exclusiva - Revizee), que possibilitaram a obtenção de algumas informações sobre os Tintinnina (Sassi et al. 1996, Galvão 2000).

Neste trabalho procurou-se conhecer a diversidade dos Tintinnina que ocorrem na área de influência do Atol das Rocas e do Arquipélago de Fernando de Noronha, Nordeste do Brasil, estudar a sua distribuição espacial na área e caracterizar alguns parâmetros que definem a estrutura da comunidade desses organismos, como diversidade e eqüitabilidade. A importância do estudo desses ciliados planctônicos justifica-se, porque eles estão entre os consumidores primários mais ativos do ambiente pelágico, podendo reduzir substanciais frações do carbono orgânico fixado pelo fitoplâncton por dia, com valores de utilização da produção primária que podem chegar a $70 \%$ (Beers \& Stewart 1970) e até mesmo a 100\% (Capriulo \& Carpenter 1983). Apesar de representarem uma fração muito pequena dos ciliados planctônicos (menos que $10 \%$ da biomassa, segundo Dolan 2000) alimentam-se ativamente de células do nanoplâncton e do picoplâncton (Capriulo et al. 1991, 2002), têm uma elevada capacidade de reciclar nutrientes in situ, devido aos seus altos ritmos metabólicos, ciclos de vida curtos e a uma biomassa expressiva em muitos ambientes marinhos, e desempenham um papel importante nas transferências energéticas do ambiente pelágico (Laval-Peuto \& Brownlee 1986, Pierce \& Turner 1994, Johansson et al. 2004), sem contar ainda que muitas espécies são excelentes bioindicadores de afloramentos de massas d'água (Balech 1972).

\section{Material e Métodos}

Foram analisadas 27 amostras de plâncton coletadas em 27 estações estabelecidas em função do programa de amostragem do Navio Oceanográfico Seward Johnson, Harbor Branch Oceanographic Institution, Cruzeiro SJ9902 (Tabela 1), durante a execução do projeto "Estudo da história natural do tubarão-limão (Negaprion brevirostris Poey, 1968), no Atol das Rocas e Fernando de Noronha", no período de 13 a 30 de março de 1999. Estas áreas encontram-se banhadas pela Corrente Sul Equatorial originada nas costas da África a partir da corrente de Benguela (Kikuchi 1994) e estão entre as principais áreas de reprodução de aves marinhas do Atlântico Sul Ocidental, além de abrigarem muitas espécies de peixes e de outros grupos de animais protegidos por leis ambientais brasileiras.

As amostras foram obtidas através de arrastos verticais desde $200 \mathrm{~m}$ até a superfície, utilizando-se para isto rede de plâncton com malha de $20 \mu \mathrm{m}$ de abertura e $30 \mathrm{~cm}$ de diâmetro de boca. $\mathrm{O}$ material coletado foi preservado em formol neutro a $4 \%$ e as análises efetuadas em microscópio invertido Zeiss (Utermöhl 1958), usando-se câmaras de sedimentação de $50 \mathrm{~mL}$. Os exemplares encontrados foram contados, medidos e identificados em nível específico.

O tratamento dos dados incluiu: (a) a análise de freqüência de ocorrência das espécies, adotando-se os seguintes critérios de classificação: muito freqüente $(>50 \%)$, freqüente $(<50 \%>30 \%)$, pouco freqüente $(>30 \%<10 \%)$ e esporádico $(<10 \%)$; (b) densidade (ind. $\mathrm{m}^{-3}$ ), calculado a partir do volume filtrado pela rede, através da fórmula $\mathrm{V}=\mathrm{r}^{2} \mathrm{~h}$, onde $\mathrm{r}^{2}$ corresponde a área da boca da rede em $\mathrm{m}^{2} \mathrm{e}$ h o comprimento da rede em m; (c) abundância relativa, adotando-se as seguintes categorias: dominante $(>50 \%)$, abundante $(>30 \%<50 \%)$, pouco abundante $(>10 \%<30 \%)$ e rara $(<10 \%)$; (d) diversidade específica, $\left(\mathrm{H}^{-1}=-\Sigma\right.$ pi. $\log _{2}$ pi) segundo Shannon (1948) e eqüitabilidade ( $\mathrm{J}=\mathrm{H}^{\prime} / \log \mathrm{S}$ ), conforme Pielou (1967).

\section{Resultados}

Das 36 espécies identificadas, 11 foram enquadradas na categoria de muito freqüentes, representando um percentual de $30,5 \%$ dos organismos identificados, e estiveram representadas por: Codonella apicata Kofoid \& Campbell, 1929, Epiplocylis undella (Ostenfeld \& Schmidt 1901), Eutintinnus apertus Kofoid \& Campbell, 1929, Eutintinnus fraknoi (Daday) Kofoid \& Campbell, 1929, Poroecus apicatus Kofoid \& Campbell, 1929, Protorhabdonella simplex (Cleve) Jörgensen, 1924, Rhabdonella amor (Cleve 1900) Brandt, 1907, R. elegans Jörgensen, 1924, Rhabdonellopsis apophysata (Cleve) Kofoid \& Campbell, 1929, Steenstrupiella gracilis (Claparède \& Laackmann, 1858) Kofoid \& Campbell, 1929 e Undella claparedei (Entz) Daday, 1887.

As espécies freqüentes contribuíram com 22,2\% de todos os Tintinnina identificados. Estiveram classificadas nesta categoria oito espécies: Amphorides quadrilineata (Claparèrede \& Laackmann, 1858), Codonellopsis tuberculata (Daday) Jörgensen, 1924, C. laciniosa (Brandt) Brandt, 1907, Coxliella sp., Dadayella ganymedes (Entz Sr., 1824), Epiplocyloides reticulata (Ostenfeld \& Schmidt, 1901), Eutintinnus stramentus Kofoid \& Campbell, 1939, Eutintinnus tubulosus (Ostenfeld) Kofoid \& Campbell, 1929.

$\mathrm{Na}$ categoria de pouco freqüente foram registradas quatro espécies, correspondendo a $11,1 \%$ dos exemplares identificados: Coxliella massuti Duran, 1953, Dictyocista mitra Haeckell, 1837, Steenstrupiella steenstrupii (Claparèrede \& Laackmann, 1858) Kofoid \& Campbell, 1929, e Undellla hyalina Daday, 1887.

A maioria das espécies (13) ocorreu em menos de 10\% das amostras, sendo consideradas esporádicas. Essas espécies contribuíram com 36,1\% dos táxons identificados e estiveram representadas por: Codonella aspera Kofoid \& Campbell, 1929, Codonella nationalis Brandt, 1906, Coxliella ampla (Jörgensen) Brandt, 1907, Dictyocista elegans lepida (Ehrenberg) Balech, 1959, Eutintinnus lususundae (Entz Sr., 1824), Metacylis mereschkowskyi Kofoid \& Campbell, 1929, M. perspicax (Hada, 1938), Parundella attenuata Kofoid \& Campbell, 1929, Undella subcaudata Jörgensen, 1924, Petalotricha ampulla (Fol, 1881) Kent, Rhabdonella henseni (Brandt 1906) Brandt, 1907, R. hydria Jörgensen, 1924 e Salpingella subconica Kofoid \& Campbell, 1929. 
Tabela 1. Posições oceanográficas das estações estudadas no Atol das Rocas (Rio Grande do Norte) e no Arquipélago de Fernando de Noronha (Pernambuco), no período de 13 a 30 de março de 1999.

Table 1. Oceanographical positions of the studied stations in the das Rocas atoll (Rio Grande do Norte) and in the Fernando de Noronha archipelago (Pernambuco), from 13 to 30 March 1999.

\begin{tabular}{|c|c|c|c|c|}
\hline Estação* & Data & Hora & Lat $S$ & Long W \\
\hline $25 \mathrm{FN}$ & $30 / 03 / 99$ & 06:03 & $03^{\circ} 53^{\prime} 00^{\prime \prime}$ & $32^{\circ} 06^{\prime} 00^{\prime \prime}$ \\
\hline $21 \mathrm{FN}$ & 29/03/99 & $22: 19$ & $03^{\circ} 52^{\prime} 00^{\prime \prime}$ & $32^{\circ} 20^{\prime} 01^{\prime \prime}$ \\
\hline $22 \mathrm{FN}$ & 29/03/99 & $23: 18$ & $03^{\circ} 51^{\prime} 95^{\prime \prime}$ & $32^{\circ} 21^{\prime} 21^{\prime \prime}$ \\
\hline $27 \mathrm{FN}$ & 30/03/99 & $15: 34$ & $03^{\circ} 52^{\prime} 00^{\prime \prime}$ & $32^{\circ} 24^{\prime} 00^{\prime \prime}$ \\
\hline $26 \mathrm{FN}$ & $30 / 03 / 99$ & 09:02 & $03^{\circ} 51^{\prime} 00^{\prime \prime}$ & $32^{\circ} 34^{\prime} 00^{\prime \prime}$ \\
\hline $20 \mathrm{FN}$ & 26/03/99 & $13: 12$ & $03^{\circ} 46^{\prime} 16^{\prime \prime}$ & $32^{\circ} 35^{\prime} 54^{\prime \prime}$ \\
\hline $23 \mathrm{FN}$ & $30 / 03 / 99$ & $01: 25$ & $03^{\circ} 53^{\prime} 00^{\prime \prime}$ & $32^{\circ} 37^{\prime} 00^{\prime \prime}$ \\
\hline $15 \mathrm{FN}$ & 26/03/99 & $01: 56$ & $03^{\circ} 51^{\prime} 97^{\prime \prime}$ & $32^{\circ} 38^{\prime} 81^{\prime \prime}$ \\
\hline $19 \mathrm{FN}$ & 26/03/99 & $11: 14$ & $03^{\circ} 46^{\prime} 51^{\prime \prime}$ & $32^{\circ} 42^{\prime} 89^{\prime \prime}$ \\
\hline $24 \mathrm{FN}$ & $30 / 03 / 99$ & $02: 58$ & $03^{\circ} 53^{\prime} 00^{\prime \prime}$ & $32^{\circ} 47^{\prime} 00^{\prime \prime}$ \\
\hline $18 \mathrm{FN}$ & 26/03/99 & 08:22 & $03^{\circ} 44^{\prime} 99^{\prime \prime}$ & $33^{\circ} 02^{\prime} 04^{\prime \prime}$ \\
\hline $17 \mathrm{FN}$ & 26/03/99 & $06 \mathrm{~h} 41$ & $03^{\circ} 45^{\prime} 02^{\prime \prime}$ & $33^{\circ} 13^{\prime} 17^{\prime \prime}$ \\
\hline $16 \mathrm{FN}$ & 26/03/99 & $04: 33$ & $03^{\circ} 46^{\prime} 03^{\prime \prime}$ & $33^{\circ} 23^{\prime} 07^{\prime \prime}$ \\
\hline $4 \mathrm{AR}$ & $14 / 03 / 99$ & $13: 49$ & $03^{\circ} 51^{\prime} 03^{\prime \prime}$ & $33^{\circ} 38^{\prime} 00^{\prime \prime}$ \\
\hline $8 \mathrm{AR}$ & $18 / 03 / 99$ & $20: 44$ & $03^{\circ} 51^{\prime} 38^{\prime \prime}$ & $33^{\circ} 38^{\prime} 70^{\prime \prime}$ \\
\hline $14 \mathrm{AR}$ & 26/03/99 & 00h59 & $03^{\circ} 51^{\prime} 00^{\prime \prime}$ & $33^{\circ} 40^{\prime} 01^{\prime \prime}$ \\
\hline $9 \mathrm{AR}$ & $18 / 03 / 99$ & $22: 24$ & $03^{\circ} 52^{\prime} 00^{\prime \prime}$ & $33^{\circ} 40^{\prime} 01^{\prime \prime}$ \\
\hline $6 \mathrm{AR}$ & $14 / 03 / 99$ & $18: 29$ & $03^{\circ} 52^{\prime} 00^{\prime \prime}$ & $33^{\circ} 41^{\prime} 00^{\prime \prime}$ \\
\hline $13 \mathrm{AR}$ & 25/03/99 & $23: 54$ & $03^{\circ} 52^{\prime} 92^{\prime \prime}$ & $33^{\circ} 41^{\prime} 80^{\prime \prime}$ \\
\hline $10 \mathrm{AR}$ & 18/03/99 & $18: 28$ & $03^{\circ} 52^{\prime} 74^{\prime \prime}$ & $33^{\circ} 41^{\prime} 92^{\prime \prime}$ \\
\hline $5 \mathrm{AR}$ & $14 / 03 / 99$ & $17: 39$ & $03^{\circ} 53^{\prime} 00^{\prime \prime}$ & $33^{\circ} 42^{\prime} 00^{\prime \prime}$ \\
\hline $7 \mathrm{AR}$ & $16 / 03 / 99$ & $23: 15$ & $03^{\circ} 49^{\prime} 00^{\prime \prime}$ & $33^{\circ} 53^{\prime} 00^{\prime \prime}$ \\
\hline $11 \mathrm{AR}$ & 18/03/99 & $19: 39$ & $03^{\circ} 49^{\prime} 96^{\prime \prime}$ & $33^{\circ} 53^{\prime} 00^{\prime \prime}$ \\
\hline $3 \mathrm{AR}$ & $13 / 03 / 99$ & $09: 27$ & $03^{\circ} 50^{\prime} 01^{\prime \prime}$ & $33^{\circ} 54^{\prime} 00^{\prime \prime}$ \\
\hline $12 \mathrm{AR}$ & $25 / 03 / 99$ & $21: 38$ & $03^{\circ} 49^{\prime} 64^{\prime \prime}$ & $33^{\circ} 54^{\prime} 00^{\prime \prime}$ \\
\hline $2 \mathrm{AR}$ & $13 / 03 / 99$ & $07: 16$ & $03^{\circ} 50^{\prime} 01^{\prime \prime}$ & $34^{\circ} 07^{\prime} 00^{\prime \prime}$ \\
\hline $1 \mathrm{AR}$ & $13 / 03 / 99$ & 01:22 & $03^{\circ} 51^{\prime} 00^{\prime \prime}$ & $34^{\circ} 44^{\prime} 00^{\prime \prime}$ \\
\hline
\end{tabular}

* As estações estão organizadas de acordo com a longitude.

* The stations are organized according to its longitude.

Apesar do número considerável de espécies identificadas, apenas E. fraknoi se mostrou quantitativamente importante, sendo considerada dominante, apresentando um percentual de $57,8 \%$ na estação 14.

As espécies abundantes foram cinco e estiveram representadas por E. apertus, $P$. apicatus, $R$. elegans, $R$. amor e $R$. apophysata. A abundância relativa de $E$. apertus e $P$. apicatus foi de $30,0 \%$ na estação 18. R. elegans apresentou 30,9\% de abundância relativa na estação 3; $R$. amor 34,1, 36,8 e 33,3\% nas estações 10, 15, e 24, respectivamente, e $R$. apophysata esteve compreendida entre 31,5 e 47,8\%, nas estações 4, 5, 6, 8, 9, 21 e 25 (Tabela 2).

Do ponto de vista quantitativo, a maior densidade foi registrada na estação 26 (122 ind. $\left.\mathrm{m}^{-3}\right)$, situada bem próxima de Fernando de Noronha. Altas densidades de indivíduos também foram registradas nas estações 1 ( 83 ind. $\mathrm{m}^{-3}$ ) e 2 ( 75 ind. $\left.\mathrm{m}^{-3}\right)$, situadas em áreas mais afastadas, e na estação 5 ( 81 ind. $\left.\mathrm{m}^{-3}\right)$, localizada bem próximo do atol da Rocas (Tabela 2).

O índice de diversidade específica, calculado com base na abundância relativa de cada táxon infragenérico, indicou que a comunidade de Tintinnina nas estações estudadas está caracterizada de um modo geral por uma diversidade variando de alta a média (Figura 1).

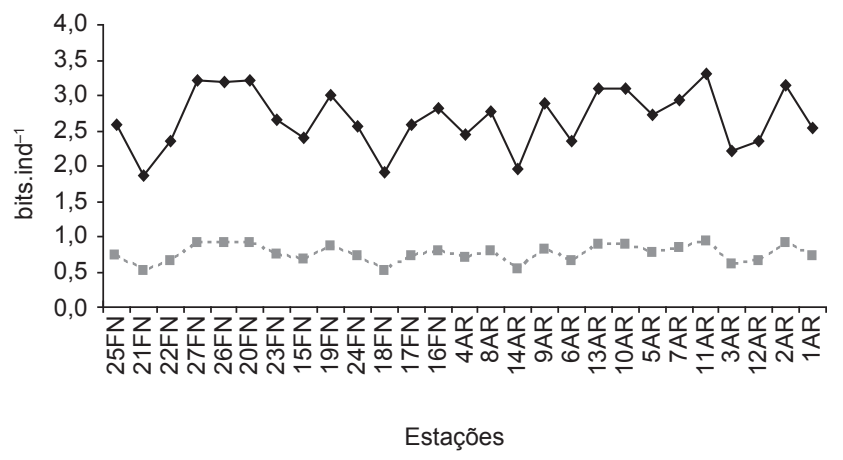

Figura 1. Variação da diversidade específica e eqüitabilidade dos Tintinnina no Atol das Rocas (RN) e Arquipélago de Fernando de Noronha (PE), no período de 13 a 30 de março de 1999.

Figure 1. Diversity specific and equitability of Tintinnina Atoll in the Rocas (Rio Grande do Norte) and Archipelago of Fernando de Noronha (Pernambuco), in March 1999.

Das 27 amostras analisadas 27,0\% apresentaram valores superiores a 3 bits.ind ${ }^{-1}$ o que caracteriza uma alta diversidade. Com diversidade média, apresentando valores acima de 2 bits.ind $^{-1}$ foram registradas 17 amostras, correspondendo a $62,9 \%$ e apenas uma amostra $(37,0 \%)$ apresentou baixa diversidade com valor inferior a 2 bits.ind $^{-1}$. Os valores mínimos e máximos, para as 27 estações, oscilaram entre 1,4 bits.ind ${ }^{-1}$ na estação 21 e 3,3 bits.ind ${ }^{-1}$ na estação 11 (Figura 1).

A eqüitabilidade variou de um mínimo de 0,59 na estação 18 e o máximo de 1,0 na estação 11 (Figura 1).

\section{Discussão}

Nas regiões oceânicas tropicais e subtropicais, o desenvolvimento da comunidade planctônica depende da disponibilidade de nutrientes inorgânicos na zona eufótica, principalmente nitrogênio, uma vez que o regime diário da luz necessária para as reações fotossintéticas é suficiente em qualquer época do ano.

No Atlântico Sul Ocidental, entre a região equatorial e a Convergência Subtropical, o estoque de nutrientes inorgânicos dissolvidos mais próximo da zona eufótica encontra-se nas camadas subsuperficiais da Água Central do Atlântico Sul, porém, a área em que o presente trabalho foi desenvolvido, apresenta-se como o setor mais pobre em plâncton, tendo em vista as condições oligotróficas da Corrente Sul Equatorial, a partir da qual se originam as correntes superficiais que influenciam na área.

Em vista desse caráter hidrográfico da região, pôde-se observar que, especialmente em relação aos Tintinnina, os resultados obtidos confirmam esta característica da água da Corrente Sul Equatorial, onde foram identificadas 36 espécies, todas elas características de águas subtropicais e tropicais, mas que também ocorrem em diversos oceanos e mares do mundo.

Das mais freqüentes, Rhabdonellopsis apophysata, Eutintinnus fraknoi, Undella claparedei, Epiplocylis undella, Rhabdonella amor e Rhabdonella elegans formam um grupo de espécies citadas por Souto (1970a, 1970b), para as águas costeiras do Brasil. Entretanto, todas essas espécies possuem lóricas do tipo hialinas e são muito comuns em águas oceânicas do Nordeste do Brasil (Sassi et al. 1996, Galvão 2000). Mas o gênero Eutintinnus, em particular, também é freqüentemente encontrado na zona costeira, onde ocorre em associação com espécies com loricas aglutinantes, particularmente do gênero Tintinnopsis (Sassi \& Melo 1989, Sassi et al. 1999, Sassi et al. 2004). Esses dois gêneros também têm sido observados com frequiência em 
Nogueira, E.M.S. et al.

Tabela 2. Abundância relativa e densidade total de Tintinnina ocorrentes no Atol das Rocas (RN) e no Arquipélago de Fernando de Noronha (PE) no período de 13 a 30 de março de 1999. $(->50 \%$ das amostras; $\odot=>30 \%<50 \%$ das amostras; $*=>10 \%<30 \%$ das amostras; $\cdot=<10 \%$ das amostras $)$.

Table 2. Relative abundance and total density of Tintinnina occurring in the das Rocas atoll (Rio Grande do Norte) and the Fernando de Noronha Archipelago (Pernambuco) in March 1999. $(\bullet=>50 \%$ of samples; $\odot=>30 \%<50 \%$ of samples; $*=>10 \%<30 \%$ of samples; $\cdot=<10 \%$ of samples).

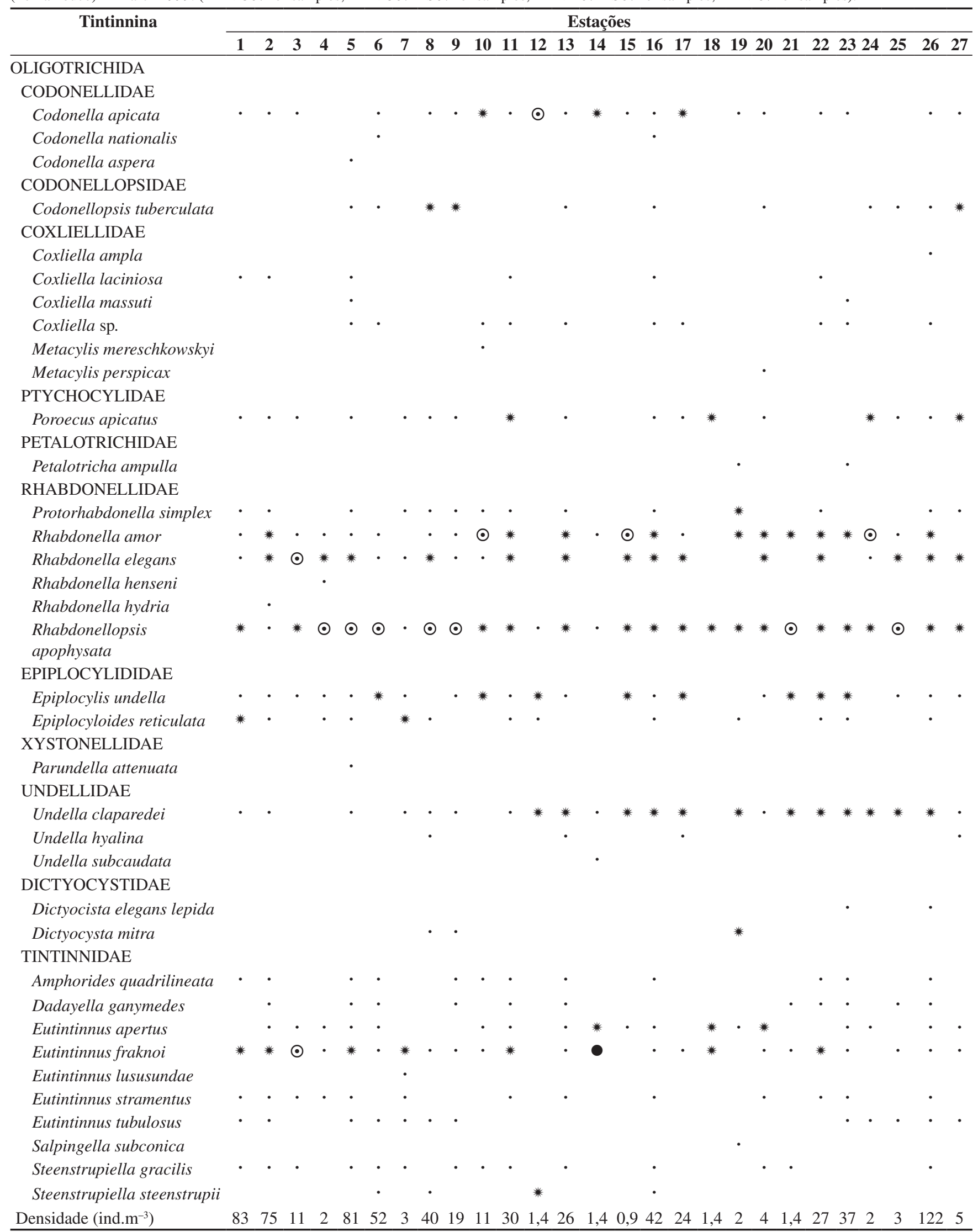


sistemas costeiros tropicais e temperados do Atlântico Norte (Sanders 1987, Verity 1987, Urrutxurtu 2004), Caribe (Gilron et al. 1991), India (Godhantaraman 2002) e Mar Mediterrâneo (Cariou et al. 1999, Modigh \& Castalado 2002).

Os dados quantitativos que foram registrados nas amostras examinadas confirmam as características oligotróficas da massa d'água local. Os valores obtidos para as densidades dos Tintinnina $\left(10^{-1}\right.$ a $10^{2}$ org. $\left.\mathrm{m}^{-3}\right)$ são similares aos que foram registrados na Costa do Estado de Pernambuco, Nordeste do Brasil (Nogueira-Paranhos 1990) e ao sul do Mar Adriático (Krsinic 1982), mas estão bem abaixo dos que foram registrados em águas mais frias do Atlântico Sul Ocidental (Thompson \& Alder 2005).

Diversos autores têm constado a existência de padrões de distribuição agregada nas populações dos Tintinnina, com formações de manchas, em amostras de superfície coletadas em áreas oceânicas (Reid 1982, Pompeu 1998). E muitas espécies de Tintinnina apresentam essa característica, agindo como estrategistas-r, reproduzindo-se muito rapidamente quando condições favoráveis existem. Assim, a persistência na área de espécies como E. fraknoi, R. apophysata, $R$. amor, R. elegans, U. claparedei e E. undella, podem igualmente apresentar essa estratégia, refletindo a sua habilidade em utilizar de forma mais eficiente os recursos alimentares disponíveis.

Segundo Capriulo (1982), a abundância e a composição dos ciliados podem estar na dependência da quantidade e tamanho do alimento disponível, tanto quanto da predação. Dados do macrozooplâncton não são disponíveis para a área, e as análises dos outros componentes do microzooplâncton, especialmente copépodos e larvas de crustáceos do microplâncton, que igualmente predam os Tintinnina, não parecem ter efeito expressivo sobre esses ciliados, pois mesmo ocorrendo de forma significativa em alguns momentos, conforme demonstrou o estudo nictemeral realizado na área (Nogueira 2000) se apresentaram com um número menor de indivíduos e mesmo quantificados juntos não ultrapassaram os números de indivíduos dos Tintinnina.

Como se constatou não se observou nenhuma área onde grandes populações de Tintinnina pudessem ser formadas; as maiores densidades foram registras em áreas mais afastadas dos bancos (estações $1 \mathrm{e}$ 2) e também em algumas estações muito próximas dos bancos (estações 5 e 22), indicando agregações das populações, possivelmente transportadas para essas áreas por processos físicos.

Os dados apresentados refletem apenas condições encontradas em águas superficiais, mas sabe-se que os Tintinnina podem apresentar distribuições superpostas de grupos de espécies, com abundâncias numéricas variáveis em cada estrato (Balech 1972, Krsinic 1982). Assim, parece plausível que grandes populações de Tintinnina sejam encontradas em águas mais profundas, onde as concentrações de clorofila-a são mais elevadas. Na região, pelo menos durante o período estudado, a termoclina mantém-se sempre abaixo de $100 \mathrm{~m}$, e as concentrações máximas de clorofila- $a$ ficam restritas a esse nível de profundidades (Cordeiro et al. 2000, relatório não publicado). Ressalta-se, porém, que a presença de espécies umbrófilas de Tintinnina coletadas na superfície durante esta pesquisa, como por exemplo, Dictyocysta mitra e U. hyalina (Balech, 1972), parece ser um indício de que algum tipo de mecanismo de ascensão de águas de níveis mais profundos pode ocorrer na área.

A propósito, os Tintinnida mostram uma ampla plasticidade alimentar (detritos, picoplâncton, bacterioplâncton, diatomáceas microplanctônicas, dinoflagelados) nos diferentes ecossistemas e/ou quando em condições de estresse de alimento (Sitran et al. 2007). Assim, se considerarmos que o fitoplâncton tem pouca expressividade na área estudada, uma vez que na superfície as concentrações de clorofila-a são muito baixas e as maiores situam-se apenas nas proximidades da termoclina e não ultrapassam 0,22 $\mu \mathrm{g} . \mathrm{L}^{-1}$ (Sampaio 1998, Cordeiro et al. 2000, dados não publicados), pode-se inferir que fontes alternativas de alimento podem estar subsidiando as cadeias alimentares pelágicas da região, possibilitando, em situações especificas, a formação de manchas de microplâncton como as que foram registradas em algumas estações. Assim, é provável que formas detritais como "mucos" e "agregados orgânicos", produzidos pelas comunidades bênticas do atol, especialmente os cnidários, possam representar um importante alimento do microzooplâncton local, nesses casos (Bowen et al. 1995). Esses agregados, citados por alguns autores como "produção paraprimária" ou "pseudoplâncton", têm sido observados com freqüência em várias regiões recifais do globo (Marshall 1965, Johannes 1967, Glynn 1973).

\section{Agradecimentos}

Agradecemos ao Dr. Ricardo de Souza Rosa, da Universidade Federal da Paraíba, pelo convite em participar do projeto 'Life History of the Lemon Shark', que nos possibilitou coletar amostras de plâncton nas imediações do Atol das Rocas e do arquipélago de Fernando de Noronha.

\section{Referências Bibliográficas}

BALECH, E. 1958. Plancton de la Campaña Antártica Argentina 1954-1955. Physis 21:75-108.

BALECH, E. 1962. Tintinnoinea y Dinoflagellata del Pacífico según material de las expediciones Norpac y Downwind del Instituto Scripps de Oceanografía. Rev. Mus. Argent. C. Nat. «B. Rivadavía», Cienc. Zool. 7(1):1-253.

BALECH, E. 1971. Microplancton del Atlantico Ecuatorial Oeste (Equalant I). Serv. Hidr. Naval. H. 654:1-103.

BALECH, E. 1972. Los Tintinnidos indicadores de afloramentos de águas (Ciliata). Physis 31(83):519-528.

BALECH, E. \& SOUTO, S. 1980. Los tintininos de la campaña oceanografica "Productividad IV". Parte I. Physis 39:1-8.

BALECH, E. \& SOUTO, S. 1981. Los tintininos de la campaña oceanográfica "Productividad" IV. Parte II. Physis 39:41-49.

KRSINIC, F. 1982. On vertical distribution of tintinnines (Ciliata, Oligotrichida, Tintinnina) in the open waters of the South Adriatic. Mar. Biol. 68(1):83-90.

BEERS, J.R. \& STEWART, G.L. 1970. Numerical abundance and estimated biomass of microzooplankton. In The ecology of the plankton off La Jolla, California, in the period April through September, 1967 (part VI) (J.D.H Strickland, ed.). Bull. Scripps Inst. Oceanogr.17:66-87.

BOWEN, S.H., LUTZ, E.V. \& AHLGREN, M.O. 1995. Dietary proteins determinants of food quality: trophic strategies compared. Ecology 76:899-907.

CAPRIULO, G.M. 1982. Feeding of field collected tintinnid microzooplankton on natural food. Mar. Biol. 71:3-86.

CAPRIULO, G.M. \& CARPENTER, E.J. 1983. Abundance, species composition and feeding impact of tintinnid micro-zooplankton in Central Long Island Sound. Mar. Ecol. Prog. Ser. 10:277-288.

CAPRIULO, G.M., SHERR, E.B. \& SHERR, B.F. 1991. Trophic behaviour and related community feeding activities of heterotrophic marine protists. In Protozoa and Their Role in Marine Processes (P. C. Reid, C. M, Turley \& P. H, Burkill, eds). Springer-Verlag, Berlin, p.219-279.

CAPRIUlO, G.M., SMITH, G., TROY, R., WIKFORS, G., PELLET, J. \& YARISH, C. 2002. The planktonic food web structure of a temperate zone estuary, and its alteration due to eutrophication. Hydrobiologia 475-476:263-333.

CARIOU, J.B., DOLAN, J.R. \& DALLOT, S. 1999. A preliminary study of tintinnid diversity in the NW Mediterranean Sea. J. Plank. Res. 21:1065-1075.

CORDEIRO, T., SASSI, R. \& NOGUEIRA, E.M.S. 2000. Report on the research project: oceanographic structure and the plankton around 
the Fernando de Noronha Chain, NE Brazil. Relatório técnico parcial. Universidade Estadual de Santa Cruz, Ilhéus, Bahia. 16p.

DOLAN, J. 2000. Tintinnid ciliate diversity in the Mediterranean Sea: Longitudinal patterns related to water column structure in late springearly summer. Aquat. Microb. Ecol. 22:20-30.

FERNANDES, L.F. 1999. Tintininos (Ciliophora - Subordem Tintinnina) de águas subantárticas e antárticas entre a Argentina e a Península Antártica ( $35^{\circ} \mathrm{S}-62^{\circ}$ S) (Novembro de 1992). Rev. Bras. Oceanogr. 47(2):155-171.

GALVÃO, T.C. 2000. Taxonomia dos Tintinnina (Protozoa- CiliophoraOligotrichida) das águas da Cadeia Norte- Brasil. Dissertação de Mestrado, Universidade Federal da Paraíba, João Pessoa.

GILRON, G.L., LYNN, D.H. \& ROFF, J.C. 1991. The annual cycle of biomass and production of tintinnine ciliates in a tropical neritic region near Kingston, Jamaica. Mar. Microb. Food Webs 5:95-113.

GLYNN, P.W. 1973. Ecology of a Caribbean coral reef. The Porites reef-flat biotope. Part II. Plankton community reef evidence for depletion. Mar. Biol. 22:1-21.

GODHANTARAMAN, N. 2002. Seasonal variations in species composition, abundance, biomass and estimated production rates of tintinnids at tropical estuarine and mangrove waters, Parangipettai, southeast coast of India. J. Mar. Sci. 36:161-171.

JOHANNES, R.E. 1967. Ecology of organic aggregates in the vicinity of a coral reef. Limnol. Oceanogr. 12:189-195.

JOHANSSON, M., OROKHOVA, E. \& LARSSON, U. 2004. Annual variability in ciliate community structure, potential prey and predators in the open northern Baltic Sea proper. J. Plank. Res. 26(1):67-80.

KIKUCHI, R.K.P. 1994. Geomorfologia, Estratigrafia e Sedimentologia do Atol das Rocas (REBIO/IBAMA/RN), Atlântico Sul Ocidental Equatorial. Dissertação de Mestrado, Universidade Federal da Bahia, Salvador.

KRSINIC, F. 1982. On vertical distribution of Tintinnines (Ciliata, Oligotrichida, Tintinnina) in the open Waters of the South Adriatic. Mar. Biol. 68:83-90.

LAVAL-PEUTO, M. \& BROWNLEE, D.C. 1986. Identification and systematics of the Tintinnina (Ciliophora): evaluation and suggestions for improvement. Annls. Inst. Oceanogr. 62(1):69-84.

MARSHALL, N. 1965. Detritus over the reef and its potential contribution to adjacent waters of Eniwetok Atoll. Ecology 46:343-344.

MODIGH, M. \& CASTALADO, S. 2002. Variability and persistence in tintinnid assemblages at a Mediterranean coastal site. Aquat. Microb. Ecol. 28:299-311.

NOGUEIRA, E.M.S. 2000. Estudo da diversidade e da estrutura da comunidade dos Tintinnina (Protozoa - Ciliophora - Oligotrichida) da região do Atol das Rocas e Arquipélago de Fernando de Noronha, Brasil. Dissertação de Mestrado, Universidade Federal da Paraíba, João Pessoa.

NOGUEIRA-PARANHOS, J.D. 1990. Taxonomia e ecologia dos Tintinnina em um trecho da plataforma continental de Pernambuco, Brasil. Dissertação de Mestrado, Universidade Federal de Pernambuco, Recife.

NOGUEIRA-PARANHOS, J.D. \& PARANAGUÁ, M.N. 1991. Estudo dos Tintinnina em um trecho da plataforma continental de Pernambuco Brasil. Anais do IV Encontro Brasileiro de Plâncton, p. 221-250.

ODUM, E.P. 1988. Ecologia. Rio de Janeiro, Guanabara. 434p.

PIELOU, E.C. 1967. The use of information theory in the study of the diversity of biological populations. Proc. 5th Berkeley Symp. Math. Stat. Probability, Univ. Calif. 4:163-177.

PIERCE, R.W. \& TURNER, J.T. 1994. Plankton studies in Buzzards Bay, Massachussetts, USA. IV. Tintinnids, 1987 to 1988. Mar. Ecol. Prog. Ser. 112:235-240.

POMPEU, M. 1998. Microzooplâncton - Tintinnina da região de Abrolhos $\left(16^{\circ}\right.$ a $21^{\circ} \mathrm{S}$ a $\left.41^{\circ} \mathrm{W}\right)$. Dissertação de Mestrado, Universidade de São Paulo, São Paulo.
REID, P.C. 1982. Patterns of spatial and temporal variability of marine planktonic Protozoa. Ann. Inst. oceanogr. 58(S):79-190.

RICKLEFS, R.E. 2003. A Economia da Natureza. 5 ed. Guanabara Koogan, Rio de Janeiro. 478p.

SAMPAIO, J.A.A. 1998. Distribuição horizontal e vertical da clorofila-a fitoplanctônica em águas da zona econômica exclusiva do nordeste brasileiro. Relatório Técnico Final. 21p.

SANDERS, R.W. 1987. Tintinnids and other microzooplankton - seasonal distributions and relationships to resources and hydrography in a Maine estuary. J. Plank. Res. 9:65-77.

SASSI, R. \& MELO, G.N. 1983. Levantamento preliminar dos Tintinoíneos (Protozoa-Oligotrichida) da Ponta dos Seixas, Paraíba, Brasil. In Encontro de Zoologia do Nordeste, 4. Resumo dos trabalhos. Maceió, p. 73.

SASSI, R. \& MELO, G.N. 1986. Tintinnina (Protozoa-CiliophoraOligotrichida) from the first Brazilian Expedition to the Antartic. An. Acad. Bra. Ciênc. 58(Supl.):63-83.

SASSI, R. \& MELO, G.N. 1989. Hyaline Tintinnina (Protozoa-CiliophoraOligotrichida) from northeast Brazilian coastal reefs. Bolm Inst. oceanogr. 37(1):59-74.

SASSI, R., LIMA, M.F. \& MELO, G.N. 1996. Composição e distribuição do microzooplâncton da Cadeia de Fernando de Noronha (Atlântico Sul Ocidental), durante o verão de 1995. In: I Workshop - REVIZEE Nordeste I. Resumo dos Trabalhos. Recife, 120p.

SASSI, R., KUTNER, M.B.B. \& POMPEU, M. 1999. First record of the ciliate Eutintinnus apertus with the attached diatom Chaetoceros tetrastichon in neritic waters of Southwestern Atlantic. Rev. Nord. Biol. 13(1/2):47-60.

SASSI, R, GALVÃO, T.C.O, LIMA, M.F. \& COSTA, C.F. 2004. Tintinnina (Protozoa-Ciliophora-Oligotrichida) found in plankton samples collected in marine coastal waters from Northeast Brazil. Arq. Ciênc. Mar. 37:15-27.

SHANNON, C.E. 1948. A mathematical theory of communication. Bol. Syst. Tech. J. 27:379-423.

SITRAN, R. BERGAMASCO, A., DECEMBRINI, F. \& GUGLIELMO, L. 2007. Temporal succession of tintinnids in the northern Ionian sea, central Mediterranean. J. Plank. Res. 29:495-508.

SOUTO, S. 1970a. Tintínnidos de la costa atlántica entre los $31^{\circ}$ y $35^{\circ}$ de latitud Sur (Uruguay y Sur de Brasil). Physis 30(80):187-208.

SOUTO, S. 1970b. Tintínnidos de la costa brasileira colectados por el Walter Herwig. Physis 30(80):209-224.

SOUTO, S. 1972. Tintínnidos subantárticos del Mar Argentino (ProtozoaCiliata). Physis A 31:451-462.

THOMPSON, G.A. \& ALDER, V.A. 2005. Patterns in tintinnid species composition and abundance in relation to hydrological conditions of the Southwestern Atlantic during austral spring. Aquat. Microb. Ecol. 40:85-101.

THOMPSON, G.A., ALDER, V.A., BOLTOVSKOY, D. \& BRANDINI, F. 1999. Abundance and biogeography of tintinnids (Ciliophora) and associated microzooplankton in the Southwestern Atlantic Ocean. J. Plank. Res. 21(7):1265-1298.

URRUTXURTU, I. 2004. Seasonal succession of tintinnids in the Nervion River Estuary, Basque Country, Spain. J. Plank. Res. 26:307-314.

UTERMÖHL, H. 1958. Zur Vervollkommung der quantitativen PhytoplanktonMethodik. Mitt. Int. Ver. Theor. Angew. Limnol. 9:1-38.

VERITY, P.G. 1987. Abundance, community composition, size distribution, and production rates of tintinnids in Narragansett Bay, Rhode Island. Est. Coast. Shelf Sci. 24:671-690. 